\title{
A placebo-controlled trial of simvastatin therapy in Smith-Lemli-Opitz syndrome
}

\author{
Christopher A. Wassif,PhD', Lisa Kratz, PhD², Susan E. Sparks, MD ${ }^{1,3}$, Courtney Wheeler, MS4, \\ Simona Bianconi, MD ${ }^{1}$, Andrea Gropman, $\mathrm{MD}^{5}$, Karim A. Calis, PharmD, $\mathrm{MPH}^{6}$, \\ Richard I. Kelley, MD, PhD7, Elaine Tierney, $\mathrm{MD}^{4}$ and Forbes D. Porter, MD, PhD ${ }^{1}$
}

\begin{abstract}
Background: Smith-Lemli-Opitz syndrome (SLOS) is a multiple malformation/cognitive impairment syndrome characterized by the accumulation of 7-dehydrocholesterol, a precursor sterol of cholesterol. Simvastatin, a 3-hydroxy-3-methylglutaryl-coenzyme A reductase inhibitor that crosses the blood-brain barrier, has been proposed for the treatment of SLOS based on in vitro and in vivo studies suggesting that simvastatin increases the expression of hypomorphic DHCR7 alleles.
\end{abstract}

Methods: Safety and efficacy of simvastatin therapy in 23 patients with mild to typical SLOS were evaluated in a randomized, doubleblind, placebo-controlled trial. The crossover trial consisted of two 12-month treatment phases separated by a 2-month washout period.

Results: No safety issues were identified in this study. Plasma dehydrocholesterol concentrations decreased significantly: $8.9 \pm 8.4 \%$ on placebo to $6.1 \pm 5.5 \%$ on simvastatin $(P<0.005)$; we observed a trend toward decreased cerebrospinal fluid dehydrocholesterol concentrations. A significant improvement $(P=0.017$, paired $t$-test) was observed on the irritability subscale of the Aberrant Behavior Checklist-C when subjects were taking simvastatin.

Conclusion: This article reports what is, to our knowledge, the first randomized, placebo-controlled trial designed to test the safety and efficacy of simvastatin therapy in SLOS. Simvastatin seems to be relatively safe in patients with SLOS, improves the serum dehydrocholesterol-to-total sterol ratio, and significantly improves irritability symptoms in patients with mild to classic SLOS.

Genet Med advance online publication 11 August 2016

Key Words: Aberrant Behavior Checklist-Community; DHCR7; randomized, placebo-controlled trial; simvastatin; Smith-Lemli-Opitz syndrome

\section{INTRODUCTION}

Smith-Lemli-Opitz syndrome (SLOS; OMIM 270400) is an autosomal recessive, multiple malformation syndrome first described by Smith, Lemli, and Opitz in 1964 (ref. 1). Nearly 30 years passed before Irons et al. ${ }^{2}$ determined the biochemical nature of the disorder. Biochemically, SLOS is characterized by the abnormal accumulation of 7-dehydrocholesterol (DHC). The gene 7-dehydrocholesterol reductase (DHCR7) encodes the enzyme that reduces 7-DHC to cholesterol. DHCR7 was independently cloned by three groups in 1998, and mutations within the gene were shown to be the molecular basis of SLOS. ${ }^{3-5}$ Impaired DHCR7 activity results in increased levels of 7-DHC and its isomer 8-dehydrocholesterol. Cholesterol levels are frequently low in SLOS patients but can be in the normal range. The incidence of SLOS has been estimated to be 1 in $25,000-60,000 .^{6-9}$

The SLOS phenotype varies broadly. At the severe end of the phenotypic spectrum, SLOS is a lethal disorder due to multiple major congenital anomalies. By contrast, at the mild end of the phenotypic spectrum, SLOS patients manifest fewer and minor physical abnormalities in combination with lesser functional cognitive and fewer behavioral deficits. Congenital anomalies are variable but can include second- and third-toe syndactyly, microcephaly, neonatal or postnatal cataracts, micrognathia, cleft palate, postaxial polydactyly, cardiac defects, pyloric stenosis, and genital malformations. ${ }^{10,11}$

Currently, most SLOS patients are treated with dietary cholesterol supplementation. Although cholesterol therapy reduces serum 7-DHC concentrations to a degree, significant amounts of 7-DHC persist even after years of therapy. ${ }^{12,13}$ Anecdotal case studies and case series support the idea that cholesterol supplementation benefits the overall well-being of SLOS patients; ${ }^{10,14-18}$ however, the effects of dietary cholesterol supplementation on cognitive or behavioral aspects of this disorder have not been reported by others ${ }^{19,20}$ or substantiated in a limited controlled trial..$^{21}$ The efficacy of dietary cholesterol supplementation is

\footnotetext{
${ }^{1}$ Section on Molecular Dysmorphology, Division of Translational Medicine, Eunice Kennedy Shriver National Institute of Child Health and Human Development, National Institutes of Health, Department of Health and Human Services, Bethesda, Maryland, USA; ${ }^{2}$ Department of Neurogenetics, Kennedy Krieger Institute, Baltimore, Maryland, USA; ${ }^{3}$ Current affiliation: Sanofi Genzyme, Cambridge, Massachusetts, USA; ${ }^{4}$ Department of Psychiatry, Kennedy Krieger Institute, Baltimore, Maryland, USA; ${ }^{5}$ epartment of Neurology, Children's National Medical Center, Center for Neuroscience and Behavioral Medicine, Washington, DC, USA; ${ }^{6}$ Office of the Clinical Director, Eunice Kennedy Shriver National Institute of Child Health and Human Development, National Institutes of Health, Department of Health and Human Services, Bethesda, Maryland, USA; ${ }^{7}$ Department of Genetics and Genomics, Boston Children’s Hospital, Boston, Massachusetts, USA. Correspondence: Forbes D. Porter (fdporter@mail.nih.gov) Or Elaine Tierney (tierney@kennedykrieger.org) 
probably limited by the inability of dietary cholesterol to cross the blood-brain barrier. ${ }^{22}$ Moreover, increased concentrations of 7-DHC or 7-DHC-derived oxysterol could have toxic effects. ${ }^{23-26}$ Specialists have hypothesized that, in patients with mild to classic SLOS, many aspects of the abnormal behavioral and cognitive phenotype could be the result of altered sterol composition in the central nervous system. Thus, interventions that ameliorate the central nervous system biochemical disturbances in SLOS are critical to understanding the pathological processes that underlie this inborn error of cholesterol synthesis and to developing effective therapies to treat the neurological deficits.

The use of 3-hydroxy-3-methylglutaryl-coenzyme A reductase inhibitors as an adjunctive therapy to dietary cholesterol supplementation was considered soon after the biochemical defect was determined, ${ }^{27}$ and several case reports with divergent outcomes were published. For example, Starck et al. ${ }^{18}$ reported adverse events in two severely affected patients with SLOS treated with simvastatin, and Jira et al. ${ }^{12,28}$ reported that simvastatin therapy reduced serum and cerebrospinal fluid (CSF) concentrations of 7-DHC and, paradoxically, increased plasma and CSF cholesterol concentrations in two SLOS patients. Although the studies were confounded by concurrent dietary cholesterol supplementation, an increase in serum cholesterol in SLOS patients treated with simvastatin was also reported in two other case reports. ${ }^{29,30}$

Expression of DHCR7 is regulated by SREBP2, which, when activated by low levels of cholesterol in the endoplasmic reticulum, increases the transcription of most genes of the cholesterol synthetic pathway. Having shown that DHCR7 expression is increased in SLOS fibroblasts treated with simvastatin, ${ }^{31}$ we hypothesized that the paradoxical increase in serum cholesterol could be the result of increased expression of a DHCR7 allele with residual enzymatic function, and we demonstrated that many DHCR7 alleles encode an enzyme with residual activity. ${ }^{31}$ Furthermore, both in vitro experiments with human fibroblasts ${ }^{31}$ and in vivo experiments using hypomorphic $D h c r 7^{\text {T93M/delta }}$ mice ${ }^{32}$ support the hypothesis that increased expression of DHCR7 alleles with residual enzymatic activity can significantly improve plasma and tissue sterol concentrations. Because residual DHCR7 activity varies among patients with SLOS, this hypothesis could explain the paradoxical increase in cholesterol in some patients and the adverse reactions observed in others.

Subsequent to the initiation of our trial, additional reports describing the use of simvastatin in SLOS were published, although none were placebo-controlled trials. Chan et al. ${ }^{33}$ reported a decrease in plasma 7-DHC but no increase in the cholesterol level in three subjects treated with up to $0.4 \mathrm{mg} /$ $\mathrm{kg} /$ day of simvastatin and a high-cholesterol diet. Szabó et al. ${ }^{30}$ reported decreased 7-DHC and increased cholesterol levels in a single subject after 2 years of $0.2 \mathrm{mg} / \mathrm{kg} /$ day simvastatin and dietary cholesterol supplementation (150-250 mg/kg/day). They also reported improved behavior and gross motor function. Roullet et al..$^{34}$ observed no change in the dehydrocholesterol (7-DHC + 8-DCH)-to-cholesterol ratio in 9 subjects with SLOS treated with an average simvastatin dose of $0.23 \mathrm{mg} /$ $\mathrm{kg} /$ day. Hass et al..$^{35}$ published a retrolective study of simvastatin treatment $(1 \mathrm{mg} / \mathrm{kg} /$ day $)$ of 15 subjects with SLOS. They found a decrease in the dehydrocholesterol-to-cholesterol ratio but no positive effects on behavior. Six of their subjects taking this dose of simvastatin had significant adverse effects (sleep disturbance, increased serum transaminase concentrations, autoagression) that resulted in the reduction or discontinuation of simvastatin therapy. None of these studies included a control group, none evaluated CSF sterols, and none included prespecified clinical outcome measures. In this article we report the findings of a placebo-controlled crossover trial of simvastatin therapy in SLOS, in which we demonstrate a significant decrease in the plasma dehydrocholesterol-to-total sterol ratio, a trend toward decreased CSF 7-DHC concentrations, and a significant decrease in irritability.

\section{MATERIALS AND METHODS}

\section{Human subjects research protections}

This study was approved by the National Institute of Child Health and Human Development Institutional Review Board. Neurocognitive testing performed at the Kennedy Krieger Institute in Baltimore, Maryland, was also approved by the Johns Hopkins Institutional Review Board. This study was registered on ClinicalTrials.gov (NCT00064792). Informed consent was obtained from parents or guardians. Because of the cognitive impairment associated with this disorder, patient assent was not feasible. The study was monitored by the National Institute of Child Health and Human Development Data Safety Monitoring Committee.

\section{Subjects}

The study was limited to individuals 4 to 18 years of age at enrollment with a biochemical diagnosis of SLOS (based on an increased level of 7-DHC). For both safety reasons and because our hypothesis assumes the presence of a DHCR7 allele with residual enzymatic function, enrollment was limited to subjects with clinically mild to typical SLOS, with an SLOS severity score ${ }^{10} \leq 30$, a diagnostic DHC-to-cholesterol ratio $\leq 1.0$, and demonstration in the subjects' fibroblasts of residual cholesterol synthesis $\geq 10 \%$ of that observed in control lines. ${ }^{31}$ Subjects were excluded if they had acute liver disease or serum transaminase concentrations more than three times normal, a history of myopathy or serum creatine phosphokinase (CPK) more than three times normal, or a weight less than $10 \mathrm{~kg}$. The accrual limit was 25 subjects, with the goal of having at least 18 subjects complete the study.

\section{Simvastatin, placebo, and cholesterol preparations}

Simvastatin was obtained from Xenos Bioresources and compounded by the National Institutes of Health Clinical Center Pharmacy Development Service at a concentration of $5 \mathrm{mg} / \mathrm{ml}$ in ORA-Plus $0.75 \mathrm{ml}$ and cherry flavor $0.25 \mathrm{ml}$. The Pharmacy Development Service also prepared a placebo that was indistinguishable from the active product in appearance and taste. The 
use of simvastatin in this research study was covered by IND 67,321 . Simvastatin was administered as a single daily dose of $0.5 \mathrm{mg} / \mathrm{kg}$ for the first 6 weeks of the trial and $1.0 \mathrm{mg} / \mathrm{kg} /$ day for the remaining 46 weeks of the trial. The maximum dose was $40 \mathrm{mg} /$ day. The dose selected for this study was based on that used by Jira et al. ${ }^{12}$ Dose adjustments for weight were made at 6,14 , and 20 months. The placebo dose matched the corresponding simvastatin volume. All subjects were maintained on dietary cholesterol supplementation $(150 \mathrm{mg} / \mathrm{kg} /$ day $)$ using a cholesterol suspension $(150 \mathrm{mg} / \mathrm{ml})$ in ORA-Plus prepared by the Pharmacy Development Service.

\section{Trial design}

The study was designed as a randomized, double-blind, placebo-controlled, crossover trial consisting of two 12-month treatment and placebo arms separated by a 2 -month washout period. Randomization was performed by the Pharmacy Development Service in blocks of four. Neither the participants nor the evaluating physicians knew the assignments. Study participants were clinically evaluated, and behavioral assessments were obtained, at baseline and 6, 12, 20, and 26 months during the study. Behavioral assessment was also obtained at 14 months. Anthropomorphic measurements were obtained at each admission. The Side Effects Profile for Exceptional Children modified with additional questions related to SLOS and simvastatin (Supplementary Information online) was used to screen participants for adverse effects. Serum/plasma was collected for sterol, biochemical, or safety testing at $0,0.5$, $1,3,9,14,14.5,15,17,23$, and 26 months. CSF was collected at 0,12 , and 26 months. Parents and guardians could elect to enroll the study participants in an open-label extension after completing the crossover trial. Data regarding adverse events observed during the open-label extension were also collected.

\section{Laboratory testing}

Clinical laboratory testing was performed by the National Institutes of Health Clinical Center Department of Laboratory Medicine. Sterol concentrations were measured by the Kennedy Krieger Institute Biochemical Genetics Laboratory. Residual cholesterol synthesis in subject fibroblasts was determined as previously reported. ${ }^{31}$

\section{Statistical evaluation}

The primary outcome measure for this study was the plasma DHC-to-total sterol ratio. Secondary outcome measures were a change in the severity of the irritability subscale of the Aberrant Behavior Checklist-Community (ABC-C) $)^{36}$ completed by the parent or primary caretaker and the assessment of overall behavioral plus cognitive improvement using the Clinician's Global Impression-Improvement scale (CGI-I) ${ }^{37}$ completed by the behavioral clinician (ET) at each follow-up visit.

The study was powered based on the primary outcome measure. Prior data on plasma DHC-to-total sterol ratios from our natural history study gave a standard deviation of 0.13 for the between-subject variability. Serial data from four subjects gave a correlation of 0.95 supporting feasibility of a crossover design and yielded an expected same-subject SD of approximately 0.04 . A power calculation indicated that, in a crossover design, we could detect a difference of 0.03 between treatment and control means at $P=0.05$, with $80 \%$ power in 18 subjects. Statistical analysis was performed using Prism 6 (GraphPad Software, La Jolla, CA). Two sided paired $t$-tests were used when data were available from both phases; otherwise, unpaired $t$-tests were used. An F-test was performed to compare variances. A $P$ value less than 0.05 was considered significant, and values between 0.05 and 0.10 were considered to indicate a trend. No adjustment was made for multiple comparisons of the prespecified primary and secondary outcome measures. Data are expressed as mean \pm SD unless otherwise noted.

\section{RESULTS}

\section{Study subjects}

Of 23 subjects with SLOS initially enrolled in this study, 22 were randomized and 18 completed the study (Figure 1). Demographics, SLOS severity, DHCR7 genotype, SLOS severity score, residual cholesterol synthesis, and baseline sterol values for all participants are provided in Table 1 . Of the 22 randomized subjects, 13 (59\%) were male and 9 were female. Age at enrollment ranged from 4.0 to 17.5 years, with a mean and median of 8.2 and 7.3 years, respectively. The SLOS severity score ranged from 6 to 28 , with a mean and median of 13.2 and 11, respectively. Fibroblast residual fractional cholesterol synthesis ranged from 0.11 to 0.76 , with a mean and median of 0.37 and 0.30 , respectively. Baseline DHC-to-sterol ratio for plasma and CSF were $9.1 \pm 7.4 \%$ and $6.7 \pm 4.0 \%$, respectively.

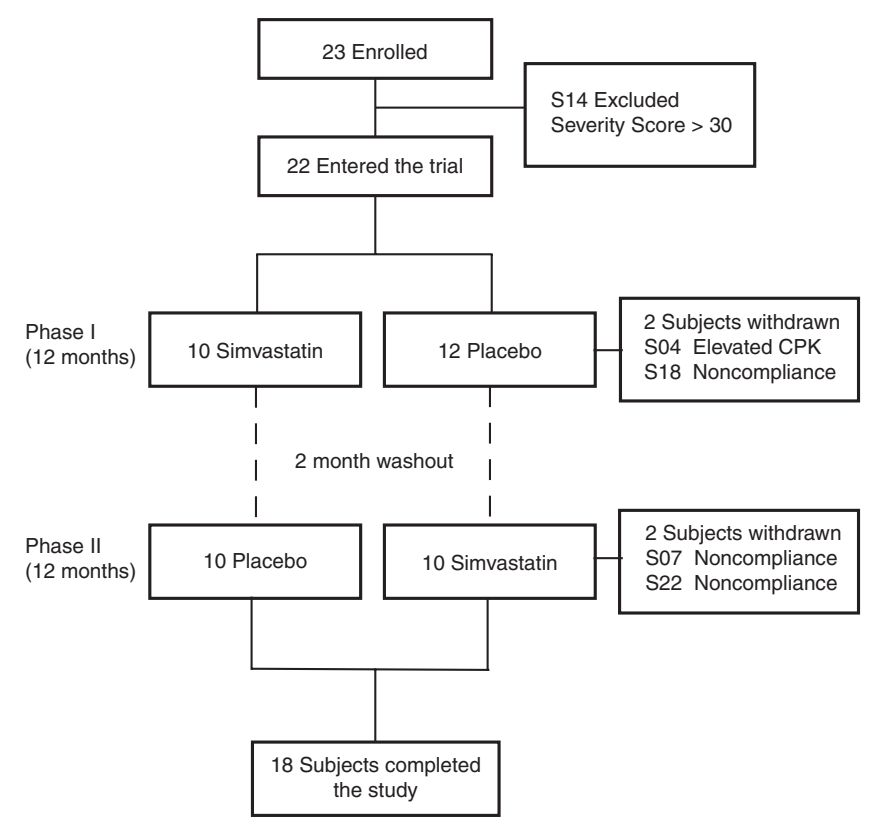

Figure 1 Study design. Subjects were randomized to either placebo or simvastatin in the first phase of this crossover trial. After 12 months in phase 1 and a 2-month washout period, subjects were switched to the alternative therapy for 12 months. One subject was excluded and four subjects were withdrawn. 


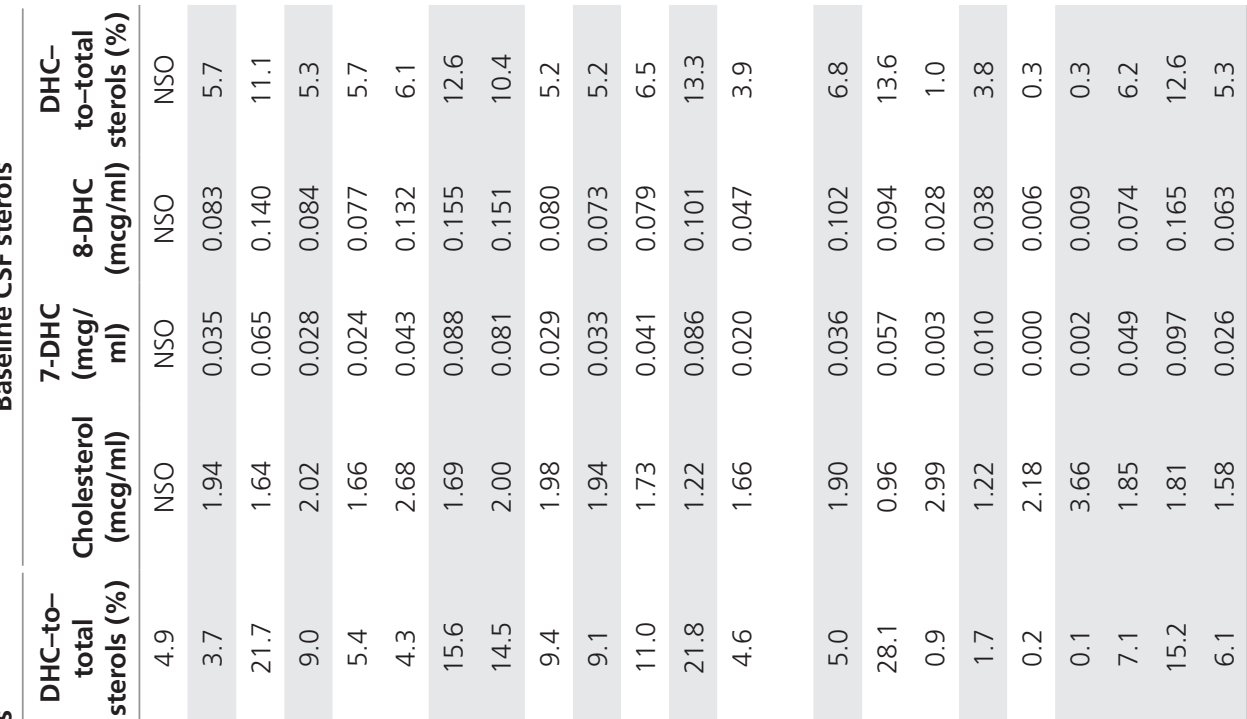

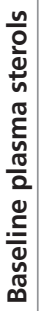

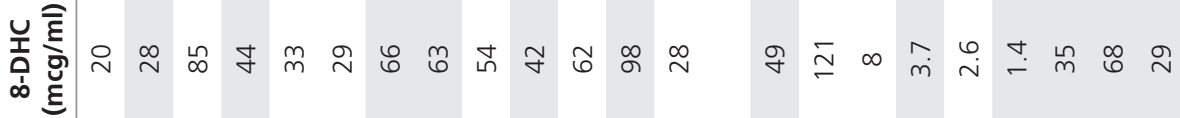

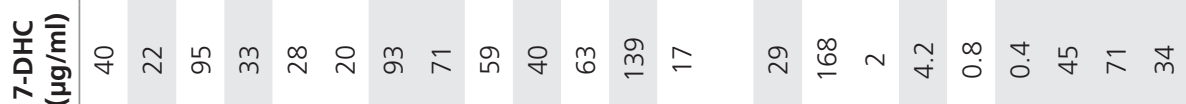

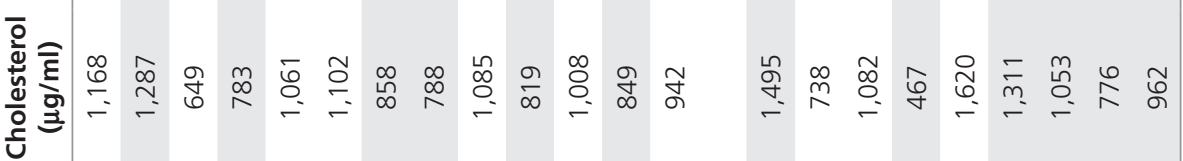

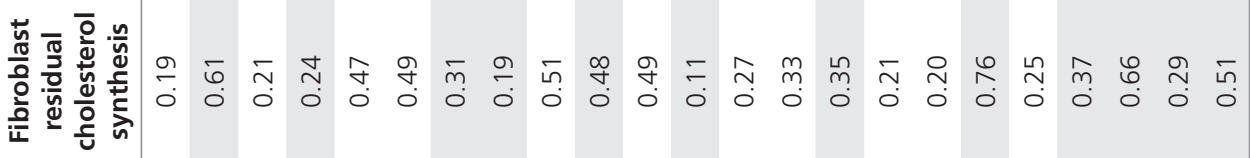

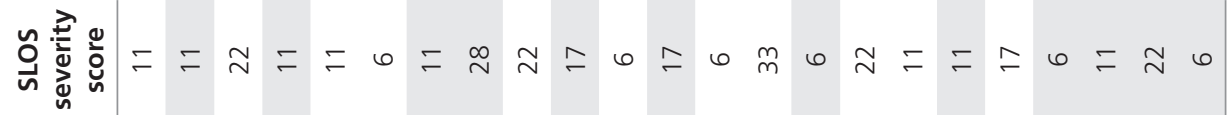

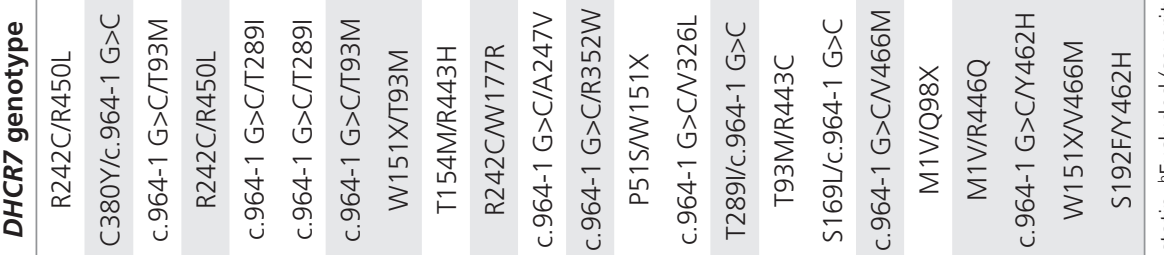

$$
\text { 离 }
$$


The plasma and CSF DHC-to-sterol ratios were highly correlated $\left(r^{2}=0.83 ; P<0.0001\right)$.

Subjects were randomized in blocks of four, with 10 and 12 subjects starting on simvastatin and placebo, respectively, for the first 12-month phase. Simvastatin therapy was initiated at $0.5 \mathrm{mg} / \mathrm{kg} / \mathrm{day}$ and increased to $1.0 \mathrm{mg} / \mathrm{kg} / \mathrm{day}$ at 6 weeks. The placebo was increased by a corresponding volume. Two subjects on placebo were withdrawn during the first phase. Subject S04 reported symptoms consistent with muscular pain and had increasing $\mathrm{CPK}$ concentrations, and thus was withdrawn at 1 month. Subject S18 was withdrawn at 6 months because of noncompliance. Twenty subjects, equally distributed between the simvastatin and placebo groups, were crossed over to the alternate study arm after a 2 -month washout period. Two subjects (S07 and S22) were withdrawn at 14 and 20 months into the second phase for noncompliance. Although both of these subjects were on simvastatin, there were no indications that noncompliance was the result of adverse drug effects. A total of 18 subjects completed both phases of the trial. There were no significant differences in gender, age, SLOS severity score, fibroblast residual cholesterol synthesis, or baseline sterol ratios between subjects who were initially randomized to either simvastatin or placebo or the 4 subjects withdrawn from the study (Supplementary Table S1 online). Because we did not observe an effect of phase order, the data were analyzed as simvastatin treatment versus placebo.

\section{Safety and adverse events}

Twenty-seven adverse events occurred in 15 subjects during the controlled part of the trial (Supplementary Table S2 online). Seven additional adverse events were noted during the openlabel extension. Five adverse events were classified as serious. Only one serious adverse event, a headache after lumbar puncture requiring extended hospitalization, occurred while taking simvastatin. No serious adverse events were considered to be drug related. Thirteen (48\%) of the adverse events occurred while taking simvastatin, and 10 of the 27 (37\%) had an infectious etiology. Eight episodes among seven subjects involved a

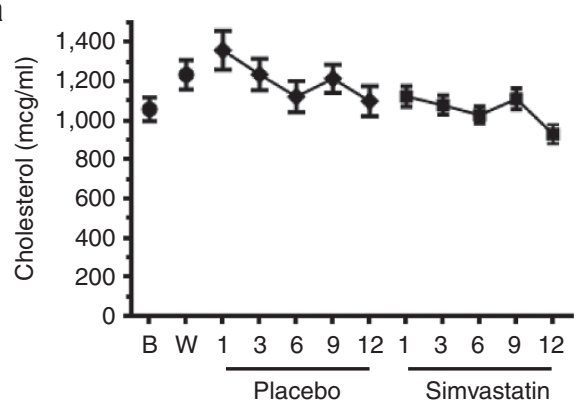

C

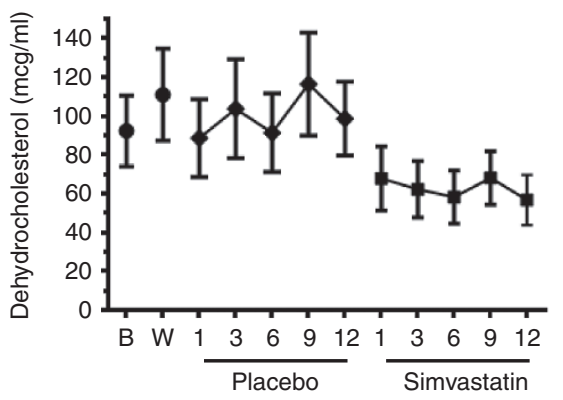

e

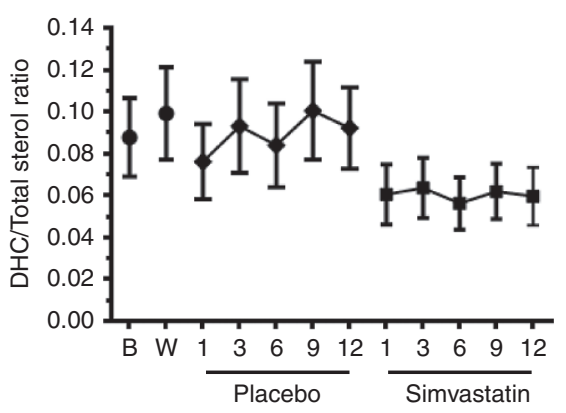

b

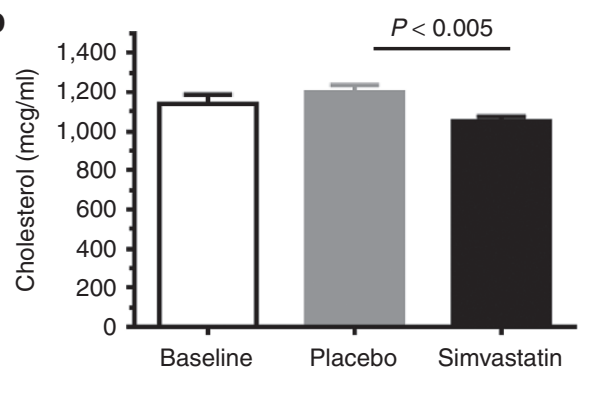

d

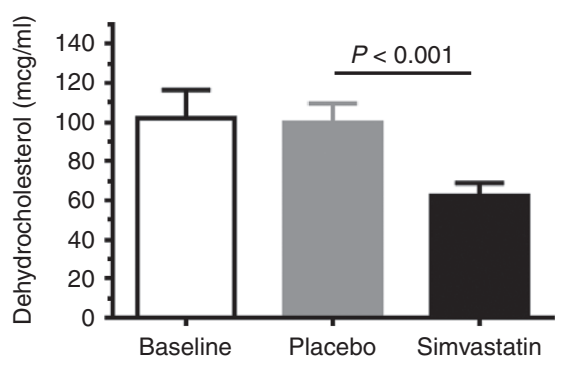

$\mathbf{f}$

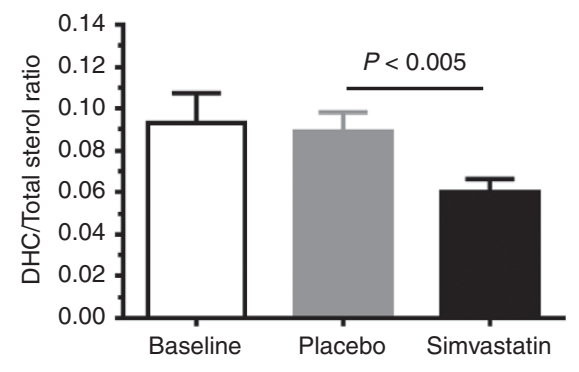

Figure 2 Plasma sterol concentrations. Cholesterol and dehydrocholesterol (DHC; 7-DHC + 8-DHC) levels were measured at baseline (B); washout (W; 14 months); and 1, 3, 6, 9, and 12 months in both the placebo and simvastatin treatment phases. Plasma cholesterol (a, b) and DHC (c, d) concentrations decreased significantly during the simvastatin phase compared with the placebo phase. The plasma DHC-to-total sterol ratio (e, f), which was the primary outcome measure of this study, also decreased significantly. Data are expressed as mean \pm SEM. 
gastrointestinal symptoms (gastroenteritis, diarrhea, vomiting, gas), with three of these (38\%) occurring when taking simvastatin. Increased aggression and self-injurious behavior was reported clinically in one subject during the open-label extension phase. Safety laboratory testing was performed at $0,0.5$, $1,3,6,9,12,14,14.5,15,17,20,23$, and 26 months. Values remained within normal ranges, and there were no significant changes in serum transaminase, alkaline phosphatase, or total bilirubin concentrations (Supplementary Figure S1 online) between the placebo and simvastatin phases. Serum CPK concentrations remained within the normal range and did not significantly differ between the placebo and simvastatin phases. The trend toward increased serum aspartate aminotransferase and $\mathrm{CPK}$ concentrations during the simvastatin phase was not considered clinically significant. Subject S04 was noted to have increasing CPK concentrations after initiation of the study. CPK concentrations in subject S04 increased from 199U/1 (normal range, 52-386 U/l) at baseline to 220 and $347 \mathrm{U} / \mathrm{l}$ at 2 and 4 weeks, respectively. Although the concentrations were still within the normal range, because the subject reported thigh pain and exhibited signs of weakness, fatigue, and worsening behavior, the investigational product was discontinued and the subject was withdrawn from the study. CPK concentrations were followed and returned to baseline after the treatment was stopped. After completion of the study, it was determined that the subject had been assigned to the placebo. No abnormalities in serum electrolytes, creatinine, glucose, inorganic phosphate, magnesium, or ionized calcium were observed. Hemoglobin and hematocrit, as well as white blood cell and platelet counts, were normal. These data suggest that patients with mild to typical SLOS are not at increased risk of liver or muscle toxicity when treated with simvastatin.

\section{Anthropomorphic parameters}

Weight and height were measured at each admission. Since subjects were not seen at 14 months, the values obtained at the 12 -month visit were used as a baseline for phase 2. Only two subjects lost weight during the trial. Subjects S12 and S16 each lost $1.2 \mathrm{~kg}$ during the placebo phase. Mean monthly weight gain during the placebo and simvastatin treatment periods were $0.20 \pm 0.16$ and $0.21 \pm 0.13 \mathrm{~kg}$, respectively (Supplementary
Figure S2A online; $n=18 ; P=0.76$, paired $t$-test). Complete data sets for height were available for 15 subjects. For two subjects, the monthly height gain could only be determined for either the simvastatin or placebo phase. Mean monthly height gain during the placebo and simvastatin treatment periods were $0.49 \pm 0.23$ and $0.42 \pm 0.20 \mathrm{~cm}$, respectively (Supplementary Figure S2B online; $n=16 ; P=0.42$, unpaired $t$-test). Our data do not support a significant change in growth parameters in subjects with mild to typical SLOS treated with simvastatin.

\section{Plasma and CSF sterol biochemistry}

Cholesterol and cholesterol precursor levels were measured at 0 (baseline); 1, 3, 6, 9, 12, and 14 months (washout); and 15, 17, 20, 23, and 26 months. Plasma sterol data are presented in Figure 2 and Supplementary Table S3 online. No significant differences were noted between plasma cholesterol concentrations $(P=$ $0.07)$, DHC concentrations $(P=0.54)$, or the DHC-to-total sterol ratio $(P=0.69)$ at baseline (time 0$)$ or 14 months after a 2 -month washout. Because changes in the plasma sterol concentrations were evident by 1 month of treatment (Figure 2a,c,e), data from all time points in each phase were combined. In contrast to previous case reports, our data do not support a paradoxical increase in plasma cholesterol concentrations with simvastatin therapy when subjects are evaluated either as a group (Figure $2 \mathbf{b}$ ) or as individuals. When the subjects were evaluated as a group, we observed a significant decrease $(P<$ 0.005 , paired $t$-test) in mean plasma cholesterol concentrations while taking simvastatin $(105 \pm 16 \mathrm{mg} / \mathrm{dl})$ compared with placebo $(120 \pm 31 \mathrm{mg} / \mathrm{dl})$. On an individual basis (Supplementary Table S3 online), a trend toward increased plasma cholesterol was only observed in one subject (S06; $P=0.05$ ). By contrast, a significant decrease in plasma cholesterol was observed in seven subjects (S04, S10, S12, S14, S15, S16, and S18). Plasma DHC concentrations were decreased in subjects with SLOS treated with simvastatin compared with placebo (Figure 2c,d). Mean DHC concentrations decreased from $106 \pm 91 \mu \mathrm{g} / \mathrm{ml}$ on placebo to $66 \pm 58 \mu \mathrm{g} / \mathrm{ml}$ on simvastatin $(P<0.001$, paired $t$-test).

The primary outcome measure of this study was the plasma DHC-to-total sterol ratio. We observed a decrease in the plasma DHC-to-total sterol ratio in subjects treated with simvastatin. The mean fraction of DHC decreased from $8.9 \pm 8.4 \%$ a

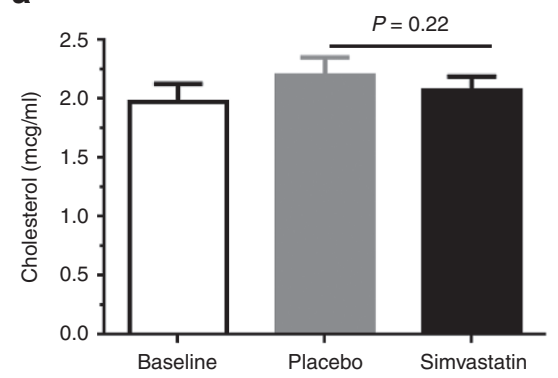

b

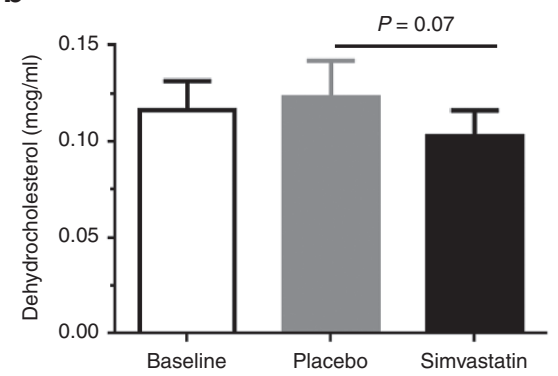

C

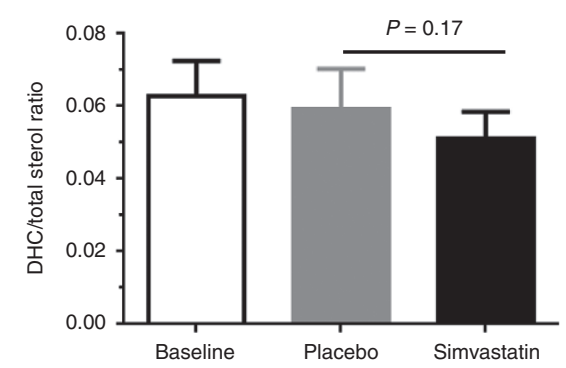

Figure 3 Cerebrospinal fluid (CSF) sterol concentrations. Neither cholesterol (a) nor the DHC-to-total sterol ratio (b) were decreased in CSF from subjects when they were treated with simvastatin compared with when they were treated with placebo. A trend toward decreased DHC (c) levels was observed during simvastatin therapy compared with placebo. Data are expressed as mean \pm SEM. 
on placebo to $6.1 \pm 5.5 \%$ on simvastatin $(P<0.005$, paired $t$-test $)$. On an individual basis, the mean fraction of DHC decreased in 17 of 18 subjects (Supplementary Table S3 online). The decrease was significant $(P<0.05)$ in 12 subjects, and 2 other subjects demonstrated a trend toward significance $(P<0.10)$.

CSF sterols were evaluated as an exploratory outcome measure (Figure 3, Supplementary Table S3 online). We observed a trend $(P=0.07$, paired $t$-test) toward decreased CSF DHC concentrations when subjects were treated with simvastatin compared with placebo. No significant difference was observed for either the CSF cholesterol level or the DHC-to-total sterol ratio in paired samples.

\section{Clinical outcome measures}

In addition to determining whether simvastatin had an effect on sterol levels, we investigated whether treatment would provide a clinical benefit by improving behavior. Our secondary outcome measures were the irritability subscale of the ABC-C and the CGI-I completed by the behavioral clinician at each follow-up visit. No significant differences were observed with the CGI-I. For the ABC-C, paired data were available for both baseline ( 0 month) and washout ( 14 months) for 13 subjects. We did not observe any significant difference $(P=0.89$, paired $t$-test) in the ABC-C irritability subscale between these two control time points (Figure 4a). However, paired comparison of the 14 subjects for whom we had data from both the placebo and simvastatin treatment arms demonstrated a significant decrease (clinical improvement; $P=0.017$ ) on the irritability subscale of the ABC-C-(Figure 4b).

\section{DISCUSSION}

This study reports the first randomized, placebo-controlled trial of simvastatin therapy in SLOS. A crossover design was used. Although crossover trials have limitations, this design facilitates the conduct of controlled trials in rare disorders, where the number of participants is limited and clinical heterogeneity is great. Our study design necessarily had several limitations. Because dietary cholesterol therapy has become the standard of care in SLOS, participants were maintained on $150 \mathrm{mg} / \mathrm{kg} /$ day of cholesterol supplementation for the duration of the study. Thus, an evaluation of simvastatin therapy independent of dietary cholesterol supplementation was precluded. Only subjects with a mild to classic SLOS phenotype were included; severely affected subjects were excluded because of safety concerns. Although apparently random, missing data may have introduced an unrecognized bias. Because of the rarity of SLOS, the necessarily small sample size in this study limited the number of outcome measures that could be evaluated. Also, the lack of generally accepted outcome measures for the characterization of abnormal behavior in SLOS necessitated the selection of clinical outcome measures without significant prior data available to validate their use.

Although difficult to conduct in rare disorders, controlled trials are essential for understanding both the safety and potential efficacy of experimental therapies. Repurposing existing drugs can expedite the development of therapies for rare and neglected diseases. However, off-label use and case reports are not substitutes for controlled trials, and the conflicting literature regarding the safety and efficacy of simvastatin therapy in SLOS highlights the limitations of case reports and uncontrolled studies.

The underlying hypothesis for this study was that treatment with simvastatin would increase DHCR7 activity by increasing the expression of DHCR7 alleles with residual enzymatic function. Increased DHCR7 activity would then decrease DHC levels relative to cholesterol and thus result in a lower DHCto-total sterol ratio. This mechanism could also potentially explain the paradoxical increases in cholesterol that have previously been reported. ${ }^{12,28}$ This potential mechanism was supported by preclinical in vitro and in vivo studies. ${ }^{31,32}$ We further hypothesized that the behavioral and cognitive aspects of SLOS result from both developmental and functional defects due to altered neuronal cholesterol composition. Although brain developmental defects are irreversible, functional defects due to altered sterol composition or neurosteroid metabolism should be amendable to therapeutic interventions that correct the biochemical defect.

Consistent with our first hypothesis, we observed a significant decrease (Figure 2) in the plasma DHC-to-total sterol ratio. This decrease was the result of a greater decrease in DHC relative to a

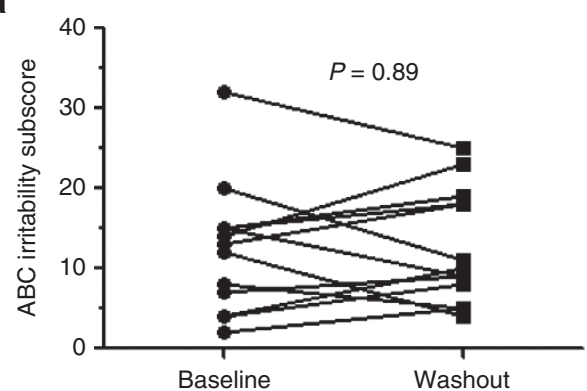

b

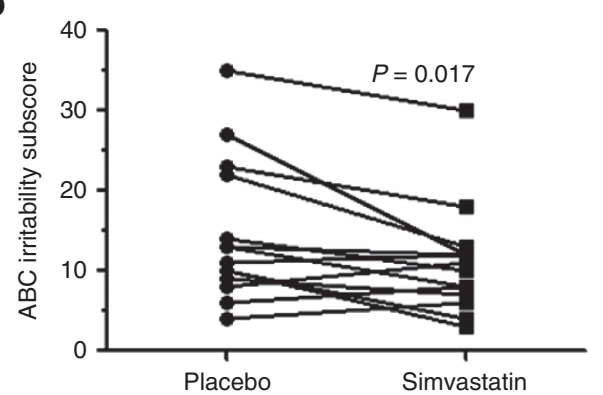

Figure 4 Simvastatin therapy reduces irritability in subjects with Smith-Lemli-Opitz syndrome. (a) Comparison of the Aberrant Behavior ChecklistCommunity (ABC-C) Irritability subscores from baseline (0 months) and washout (14 months). No significant differences were noted. (b) Paired evaluation of data from the 14 subjects for whom we had data from both the placebo and simvastatin treatment arms demonstrated a significant improvement in the ABC-C irritability subscale $(P=0.017$, paired $t$-test $)$. 


\section{ORIGINAL RESEARCH ARTICLE}

that of cholesterol. This is consistent with the data reported by Haas et al..$^{35}$ in their retrospective study. Our data do not support a concomitant increase in plasma cholesterol concentrations. For CSF sterol concentrations, which were an exploratory outcome measure in this trial, we observed a trend toward decreased DHC concentrations in CSF (Figure 3), although this was not reflected in the CSF DHC-to-total sterol ratio. CSF sterol concentrations were measured under the assumption that they might reflect synaptic cholesterol levels, but the validity of this assumption is not known. Nonetheless, this result is consistent with our observation of a disproportionate decrease in plasma 7-DHC concentrations with simvastatin therapy. It is plausible that a decrease in 7-DHC levels could have a beneficial effect on central nervous system function in SLOS. In vitro and in vivo preclinical studies supported the idea that 7-DHC or 7-DHC metabolites are toxic. 7-DHC alters the physiochemical properties, protein composition, and signaling functions of cellular membranes (reviewed in ref. 25). More recently, Francis et al..$^{26}$ showed that 7-DHC disrupts $\mathrm{Wnt} / \beta$-catenin signaling, and $\mathrm{Xu}$ et al. ${ }^{23,24}$ showed that 7 -DHCderived oxysterols, in particular $3 \beta, 5 \alpha$-dihydroxycholest-7-en-6one, accumulate in SLOS and probably have toxic effects. Thus reduction of 7-DHC or 7-DHC metabolite levels could ameliorate abnormal SLOS behaviors.

In this study we also evaluated the potential of simvastatin to alter specific aspects of the SLOS behavioral phenotype. Our secondary outcome measures were the CGI-I and ABC-C irritability scores. Although we observed no significant effect on the CGI-I, we did observe significant improvement in the ABC-C irritability score (Figure 4). This article therefore represents the first controlled study to demonstrate improved behavior in subjects with SLOS in response to a therapeutic intervention. The CGI-I is an assessment that seeks to ascertain physicians' impressions of global improvement in both cognition and behavior. The ABC-C was developed as an outcome measure for pharmacological trials including people with developmental disabilities. The ABC-C is completed by a parent or guardian. The 15-item irritability subscale includes questions about aggression, self-injury, tantrums, agitation, and unstable mood on a scale of 0 to 45 . Although we cannot directly demonstrate improved synaptic sterol composition, we were able to demonstrate changes in plasma and CSF 7-DHC concentrations consistent with the hypothesis that therapies correcting CNS sterol composition might improve behavior in SLOS and thus have a clinical benefit. Alternatively, the reduction in 7-DHC levels could have led to a reduction in 7-DHC-derived metabolites, with adverse biological effects. The small size of this study precludes the statistical evaluation of multiple neurocognitive outcome measures, and thus the evidence for clinical improvement is limited. However, the fact that we observed a significant improvement on the irritability subscale of the ABC-C provides a proof of principle for the continued development of drugs that can increase DHCR7 activity and decrease 7-DHC levels in individuals with SLOS. DHCR7 activity could be enhanced by drugs that either increase DHCR7 expression or stabilize the mutant DHCR7 protein with residual enzymatic activity.
Although increased aggression and self-injurious behavior were reported in one subject during the open-label extension phase of the study, we did not observe any consistent, treatmentlimiting adverse effects or laboratory abnormalities in subjects with SLOS treated with simvastatin, nor did we observe sleep disturbances, increased aggression, or self-injurious behavior during the controlled part of the study. We also observe no significant alteration in growth. This study supports the hypothesis that simvastatin therapy can be safely used in patients with mild to classic SLOS. However, caution is still warranted with regard to the long-term safety of simvastatin in these patients. We are aware of one subject (S23) who developed cataracts while taking off-label simvastatin therapy after this trial ended. Postnatal development of cataracts in SLOS is rare after infancy.

In summary, this study represents the first controlled trial of simvastatin therapy in SLOS and the first controlled trial demonstrating the potential of drug therapy to modulate sterol composition and to improve behavior in SLOS. We have established that treatment with simvastatin is relatively safe, can decrease DHC levels, and can improve at least one aspect of the behavioral phenotype. These data support continued efforts to identify and rigorously evaluate potential therapies that may have clinically meaningful benefits for patients with SLOS.

\section{SUPPLEMENTARY MATERIAL}

Supplementary material is linked to the online version of the paper at http://www.nature.com/gim

\section{ACKNOWLEDGMENTS}

This study was supported by the intramural research program of the Eunice Kennedy Shriver National Institute of Child Health and Human Development, by Autism Speaks, and by the Johns Hopkins Institute for Clinical and Translational Research (ICTR), which is funded in part by grant UL1 TR 001079 from the National Center for Advancing Translational Sciences (NCATS), which is a component of the National Institutes of Health $(\mathrm{NIH})$, and the $\mathrm{NIH}$ Roadmap for Medical Research. The authors acknowledge clinical and supportive assistance by Halima Goodwin, Sandra Conley, Irena Bukelis, Geeta Sarphare, and Diane Lanham, as well as the laboratory assistance by Cynthia Toth. We also thank Judith Starling and George Grimes from the NIH Clinical Center Pharmaceutical Development Service for their assistance in the preparation of the simvastatin and placebo suspensions. We offer our greatest thanks to the individuals with SLOS and their family members who participated in this clinical study.

\section{DISCLOSURE}

The authors declare no conflict of interest.

\section{REFERENCES}

1. Smith DW, Lemli L, Opitz JM. A newly recognized syndrome of multiple congenital anomalies. J Pediatr 1964;64:210-217.

2. Irons M, Elias ER, Salen G, Tint GS, Batta AK. Defective cholesterol biosynthesis in Smith-Lemli-Opitz syndrome. Lancet 1993;341:1414.

3. Fitzky BU, Witsch-Baumgartner M, Erdel M, et al. Mutations in the Delta7-sterol reductase gene in patients with the Smith-Lemli-Opitz syndrome. Proc Natl Acad Sci USA 1998;95:8181-8186. 
4. Wassif CA, Maslen C, Kachilele-Linjewile S, et al. Mutations in the human sterol delta7-reductase gene at 11q12-13 cause Smith-Lemli-Opitz syndrome. Am J Hum Genet 1998;63:55-62.

5. Waterham HR, Wijburg FA, Hennekam RC, et al. Smith-Lemli-Opitz syndrome is caused by mutations in the 7-dehydrocholesterol reductase gene. Am J Hum Genet 1998;63:329-338.

6. Ciara E, Popowska E, Piekutowska-Abramczuk D, et al. SLOS carrier frequency in Poland as determined by screening for Trp151X and Val326Leu DHCR7 mutations. Eur J Med Genet 2006;49:499-504.

7. Cross JL, Iben J, Simpson CL, et al. Determination of the allelic frequency in Smith-Lemli-Opitz syndrome by analysis of massively parallel sequencing data sets. Clin Genet 2015;87:570-575.

8. Kelley RI. A new face for an old syndrome. Am J Med Genet 1997;68: 251-256.

9. Nowaczyk MJ, Waye JS, Douketis JD. DHCR7 mutation carrier rates and prevalence of the RSH/Smith-Lemli-Opitz syndrome: where are the patients? Am J Med Genet A 2006;140:2057-2062.

10. Kelley RI, Hennekam RC. The Smith-Lemli-Opitz syndrome. J Med Genet 2000;37:321-335.

11. Porter FD. Smith-Lemli-Opitz syndrome: pathogenesis, diagnosis and management. Eur J Hum Genet 2008; 16:535-541.

12. Jira PE, Wevers RA, de Jong J, et al. Simvastatin. A new therapeutic approach for Smith-Lemli-Opitz syndrome. J Lipid Res 2000;41:1339-1346.

13. Merkens LS, Connor WE, Linck LM, Lin DS, Flavell DP, Steiner RD. Effects of dietary cholesterol on plasma lipoproteins in Smith-Lemli-Opitz syndrome. Pediatr Res 2004;56:726-732.

14. Elias ER, Irons MB, Hurley AD, Tint GS, Salen G. Clinical effects of cholesterol supplementation in six patients with the Smith-Lemli-Opitz syndrome (SLOS). Am J Med Genet 1997;68:305-310.

15. Irons M, Elias ER, Abuelo D, et al. Treatment of Smith-Lemli-Opitz syndrome: results of a multicenter trial. Am J Med Genet 1997;68:311-314.

16. Linck LM, Lin DS, Flavell D, Connor WE, Steiner RD. Cholesterol supplementation with egg yolk increases plasma cholesterol and decreases plasma 7-dehydrocholesterol in Smith-Lemli-Opitz syndrome. Am J Med Genet 2000;93:360-365.

17. Nwokoro NA, Mulvihill JJ. Cholesterol and bile acid replacement therapy in children and adults with Smith-Lemli-Opitz (SLO/RSH) syndrome. Am J Med Genet 1997;68:315-321.

18. Starck L, Björkhem I, Ritzén EM, Nilsson BY, von Döbeln U. Beneficial effects of dietary supplementation in a disorder with defective synthesis of cholesterol. A case report of a girl with Smith-Lemli-Opitz syndrome, polyneuropathy and precocious puberty. Acta Paediatr 1999;88:729-733.

19. Abuelo DN. Cholesterol supplementation in Smith-Lemli-Opitz syndrome. Am J Med Genet 1998;78:378-380.

20. Sikora DM, Ruggiero M, Petit-Kekel K, Merkens LS, Connor WE, Steiner RD Cholesterol supplementation does not improve developmental progress in Smith-Lemli-Opitz syndrome. J Pediatr 2004;144:783-791.
21. Tierney E, Conley SK, Goodwin H, Porter FD. Analysis of short-term behavioral effects of dietary cholesterol supplementation in Smith-Lemli-Opitz syndrome. Am J Med Genet A 2010;152A:91-95.

22. Dietschy JM. Central nervous system: cholesterol turnover, brain development and neurodegeneration. Biol Chem 2009;390:287-293.

23. Xu L, Korade Z, Rosado DA Jr, Liu W, Lamberson CR, Porter NA. An oxysterol biomarker for 7-dehydrocholesterol oxidation in cell/mouse models for SmithLemli-Opitz syndrome. J Lipid Res 2011;52:1222-1233.

24. Xu L, Sheflin LG, Porter NA, Fliesler SJ. 7-Dehydrocholesterol-derived oxysterols and retinal degeneration in a rat model of Smith-Lemli-Opitz syndrome. Biochim Biophys Acta 2012;1821:877-883.

25. Porter FD, Herman GE. Malformation syndromes caused by disorders of cholesterol synthesis. J Lipid Res 2011;52:6-34

26. Francis KRT, Xin Y, O'Halloran PE, et al. Modeling Smith-Lemli-Opitz syndrome with iPS cells reveals a causal role for Wnt/ $\beta$-catenin defects in neuronal cholesterol synthesis phenotypes. Nat Med 2016:22:388-96.

27. Irons M, Elias ER, Tint GS, et al. Abnormal cholesterol metabolism in the SmithLemli-Opitz syndrome: report of clinical and biochemical findings in four patients and treatment in one patient. Am J Med Genet 1994;50:347-352.

28. Jira P, Wevers R, de Jong J, Rubio-Gozalbo E, Smeitink J. New treatment strategy for Smith-Lemli-Opitz syndrome. Lancet 1997;349:1222.

29. Oberthür A, Heller R, Vogel M, et al. [Smith-Lemli-Opitz syndrome-case report, diagnostics and therapeutic options]. Z Geburtshilfe Neonatol 2009;213: 210-214.

30. Szabó GP, Oláh AV, Kozak L, et al. A patient with Smith-Lemli-Opitz syndrome: novel mutation of the DHCR7 gene and effects of therapy with simvastatin and cholesterol supplement. Eur J Pediatr 2010;169:121-123.

31. Wassif CA, Krakowiak PA, Wright BS, et al. Residual cholesterol synthesis and simvastatin induction of cholesterol synthesis in Smith-Lemli-Opitz syndrome fibroblasts. Mol Genet Metab 2005:85:96-107.

32. Correa-Cerro LS, Wassif CA, Kratz L, et al. Development and characterization of a hypomorphic Smith-Lemli-Opitz syndrome mouse model and efficacy of simvastatin therapy. Hum Mol Genet 2006;15:839-851.

33. Chan YM, Merkens LS, Connor WE, et al. Effects of dietary cholesterol and simvastatin on cholesterol synthesis in Smith-Lemli-Opitz syndrome. Pediatr Res 2009;65:681-685.

34. Roullet JB, Merkens LS, Pappu AS, et al. No evidence for mevalonate shunting in moderately affected children with Smith-Lemli-Opitz syndrome. J Inherit Metab Dis 2012;35:859-869.

35. Haas D, Garbade SF, Vohwinkel C, et al. Effects of cholesterol and simvastatin treatment in patients with Smith-Lemli-Opitz syndrome (SLOS). J Inherit Metab Dis 2007;30:375-387.

36. Marshburn EC, Aman MG. Factor validity and norms for the aberrant behavior checklist in a community sample of children with mental retardation. J Autism Dev Disord 1992:22:357-373.

37. Guy W. ECDEU Assessment Manual for Psychopharmacology, Revised. National Institute of Mental Health: Rockville, MD; 1976. 